\title{
SOME EFFECTS OF INTRINSIC CYCLIC LOADING IN SATURATED SANDS
}

\author{
ANDrZeJ SAWICKI, JACEK MiercZYŃSKi \\ Institute of Hydro-Engineering, IBW PAN, Gdańsk-Oliwa, Poland \\ e-mail:as@ibwpan.gda.pl;mier@ibwpan.gda.pl
}

\begin{abstract}
This paper presents original results of triaxial experiments performed at a cyclically changed pore pressure (intrinsic cyclic loading). The results display unusual behaviour of saturated sand subjected to such atypical loading. Some features of this behaviour are shown, including the following effects: static liquefaction of initially contractive sand, apparent creep of initially dilative sand, initial anisotropy, etc. Some differences between the classical geotechnical understanding of the effects of external cyclic loadings (such as earthquakes) and those caused by intrinsic cyclic loadings (such as wave-induced pressures) are discussed.
\end{abstract}

Keywords: saturated sand, cyclic loading, pore pressure, triaxial tests, deformations

\section{Introduction}

Cyclic loads in granular soils cause some interesting effects such as compaction, degradation of soil macroscopic mechanical properties and liquefaction. These phenomena have catastrophic consequences for infrastructure such as excessive settlements of the subsoil and subsequent destruction of buildings, the sinking of structures in liquefied soil, massive landslides, etc. The above phenomena are caused by external excitations such as earthquakes, machine vibrations, traffic, etc. These external loads lead, among others, to such phenomena as pore-pressure generation and subsequent liquefaction of saturated soils. The sequence of events can be illustrated by the following pattern: external excitation $\rightarrow$ pore-pressure generation $\rightarrow$ degradation of soil macroscopic properties $\rightarrow$ liquefaction $\rightarrow$ practical consequences of liquefaction. Geotechnical research is based mainly on the above pattern. For example, during triaxial experiments under undrained conditions, external stresses are controlled, and the excess porepressure is measured until liquefaction. The phenomenon of pore-pressure generation should be described by a separate constitutive equation, as the above phenomena cannot be described by classical models that have been developed for centuries in mechanics of materials. The above problems were the subject of extensive research in the past decades, for references see Sawicki and Mierczyński (2006), Lade and Yamamuro (1999) or Jefferies and Been (2006).

However, there is also another approach to this problem, represented by marine engineers, who focus on wave-induced pore-pressures in the seabed. In order to describe this phenomenon, they use various classical models of porous media, which however lead to different results. A short state-of-the-art is presented in Sawicki and Mierczyński (2006), see also Jeng (2013). The classical standard in marine engineering was established by Yamamoto et al. (1978). Some shortcomings of their approach were discovered by Sawicki and Staroszczyk (2008), who pointed out that Yamamoto et al. (1978) did not include plastic effects, which leads to a statically inadmissible stress field. Wave-induced pore pressures can be regarded as intrinsic cyclic loading, unlike such external excitations as earthquakes, because they influence soil behaviour directly. The corresponding pattern is the following: intrinsic excitation (pore-pressure changes) $\rightarrow$ possible changes of the effective stress state $\left(K_{0}\right) \rightarrow$ other consequences, such as failures, etc. 
The above problem was noted by the authors of the present paper, see Sawicki (2013). The first idea was to examine experimentally the influence of intrinsic cyclic loading on the behaviour of saturated sand. Some experiments were performed in the cyclic triaxial apparatus, and interesting effects of intrinsic cyclic loading were observed. This paper describes these unconventional experiments in which saturated soil samples were subjected to intrinsic cyclic loading in the form of controlled pore-pressure changes, and the resulting deformations of soil samples were measured. The results of these experiments are presented and discussed here.

The object of present investigations is to build bridges between the geotechnical and marine engineering on the basis of understanding liquefaction phenomenon. That is why two different kinds of excitations leading to liquefaction have been distinguished, designated as the external and intrinsic loading, respectively.

The results obtained are important for interpretation of experimental results, related to the problems of seabed mechanics, and particularly the seabed behaviour due to actions of water waves. Perhaps, the results presented may find some other applications in geotechnical engineering and geology related to the exploration of underground resources, where some injections may be cyclically introduced.

\section{Description of experiments}

The experiments were performed in the Enel-Hydro triaxial apparatus equipped with local gauges for the measurement of horizontal and vertical strains, see Fig. 1. The "Skarpa" model sand was used in the experiments. The basic characteristics of this sand are the following: $D_{50}=0.42 \mathrm{~mm}$, $C_{u}=2.5, G=2.65, e_{\max }=0.677, e_{\min }=0.432$. The angles of internal friction for initially loose and dense sand are $\varphi=31^{\circ}$ and $41^{\circ}$, respectively. The basic aim of the experiments was to study the behaviour of saturated soil subjected to different effective stress paths corresponding to the assumed pore-pressure changes, Fig. 2.

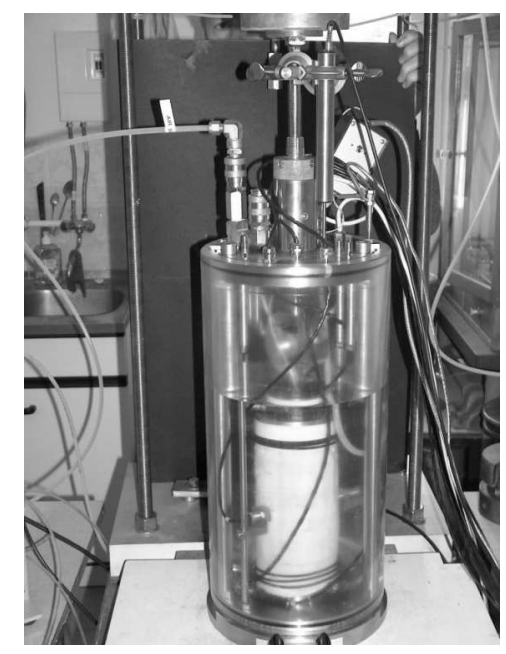

Fig. 1. The Enel-Hydro cyclic triaxial apparatus

The Enel-Hydro apparatus is equipped with the system controlling the air pressure, which forces the vertical load. This system has been modified in order to change the cyclic changes of air pressure into cyclic changes of pore water pressure. The pore water, in the drained conditions, can flow through the top and bottom bases of the soil specimen. Recall that during the experiments, the total stresses as well as the pore pressure are controlled. The vertical and horizontal strains are measured. 


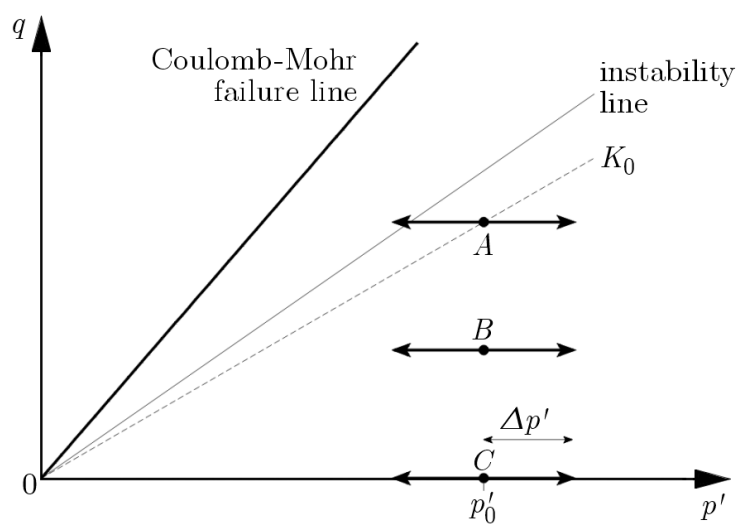

Fig. 2. The effective stress paths followed during cyclic pore-pressure changes (intrinsic cyclic loading). $A, B, C$ - initial stress states, $\Delta p^{\prime}$ - amplitude of mean effective stress changes

\section{Basic definitions}

The effective stress paths correspond to different regimes of the soil behavior. The role of instability line is displayed. The initial state of the soil and the effective stress changes define this behavior. The most important point is whether the effective stress path crosses the instability line or not.

During the experiments, the total stresses $\sigma_{z}$ (vertical) and $\sigma_{x}$ (horizontal) were kept constant, and the pore pressure $u$ was harmonically changed according to the formula

$$
u=u_{0}+\Delta u \sin 2 \pi f t
$$

where $u_{0}$ is the initial pore pressure, $\Delta u$-cyclic pore-pressure amplitude, $t$ - time. The frequency of cyclic loading was $f=0.1 \mathrm{~Hz}$.

In the description of the experiments, invariant effective stress measures are applied, i.e. the mean effective stress

$$
p^{\prime}=\frac{1}{3}\left(\sigma_{z}^{\prime}+2 \sigma_{x}^{\prime}\right)
$$

and the stress deviator

$$
q=\sigma_{z}^{\prime}-\sigma_{x}^{\prime}=\sigma_{z}-\sigma_{x}
$$

where

$$
\sigma_{z}^{\prime}=\sigma_{z}-u \quad \sigma_{x}^{\prime}=\sigma_{x}-u
$$

are normal effective stresses.

During the experiments, the mean effective stress is changed according to

$$
\Delta p^{\prime}=-\Delta u
$$

These harmonic oscillations are imposed on the initial mean effective stress $p_{0}^{\prime}=$ const in each experiment, see Fig. 2. Note that the deviatoric stress $q$ is also constant during a particular test.

During the experiments, the vertical $\varepsilon_{z}$ and horizontal $\varepsilon_{x}$ strains are recorded, but the experimental results are analyzed mainly with strain invariants, such as the volumetric strain

$$
\varepsilon_{v}=\varepsilon_{z}+2 \varepsilon_{x}
$$


and the deviatoric strain

$$
\varepsilon_{q}=\frac{2}{3}\left(\varepsilon_{z}-\varepsilon_{x}\right)
$$

Some comments regarding the effective stress space are necessary, see Fig. 2. There are two important objects in this space, namely the Coulomb-Mohr failure line and the instability line. In the case of triaxial investigations, the Coulomb-Mohr failure condition takes the following form

$$
q=\frac{6 \sin \varphi}{3-\sin \varphi} p^{\prime}=\eta^{\prime \prime} p^{\prime}
$$

The instability line corresponds to specific behaviour of granular soils in various situations. For example, in the case of undrained shearing of saturated sand, the instability line corresponds to the maximum shear stress that can be supported by initially contractive sand before its static liquefaction, see Sawicki (2011). The instability line also plays an important role in the description of the experiments presented in this paper.

Note that exactness of experimental results and their repeatability are almost a random process. It is not possible to build, for the same set of grains, two identical samples. Therefore, the mechanical properties of soils can be treated only as averages. A respective study was presented by Sawicki and Chybicki (2009).

\section{Instanteneous liquefaction of initially contractive sand}

The experiment has been performed on initially contractive sand. The initial stress state corresponds to point $A$ in Fig. 2, close to the instability line (deviatoric stress $q=77 \mathrm{kPa}$, mean effective stress $p_{0}^{\prime}=115 \mathrm{kPa}$, amplitude of cyclic changes $\left.\Delta p^{\prime}=35 \mathrm{kPa}\right)$. During the first cycle of pore-pressure changes, the mean effective stress initially increased (because of a decrease in the pore pressure, path $A a$ in Fig. 3) and then, in the second half of the cycle, decreased (because of an increase in the pore pressure, path $b c$ in Fig. 3). On reaching the instability line, the stress deviator rapidly drops, which is similar to the behaviour of soil during static liquefaction.

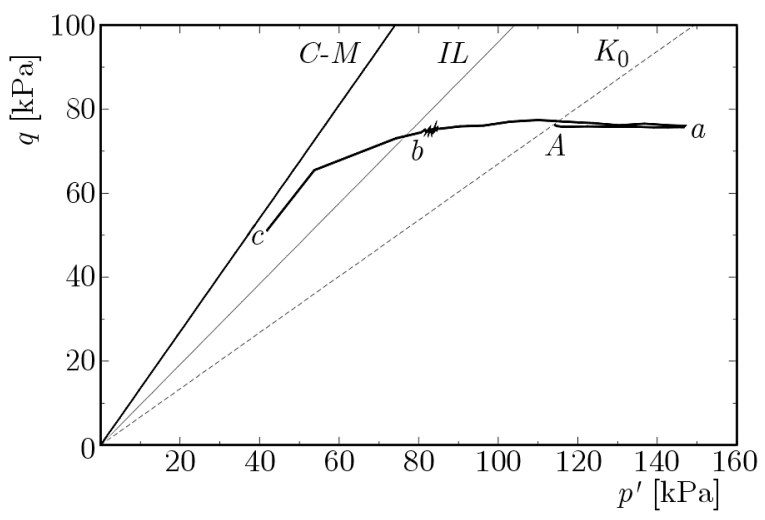

Fig. 3. Static liquefaction due to cyclic pore-pressure changes. The initial state (point $A$ ) is close to the instability line

The behaviour shown in Fig. 3 is the same as that during the undrained "spherical unloading" of initially contractive saturated sand (path $a b c$ ), see Sawicki and Świdziński (2010b). It will be shown in the next section that the behaviour of initially dilative sand is different, as the phenomenon of static liquefaction does not take place for similar effective stress paths. Recall that "geotechnical" experiments are performed under real undrained conditions under which 
pore-pressure changes are caused by external excitations. The experiments described in this paper are different because the forced pore-pressure changes (intrinsic excitation) influence the overall soil behaviour.

\section{Apparent deviatoric creep of saturated sand}

Creep is a physical phenomenon characterized by increasing deformations of materials under constant loads. This phenomenon is related to viscous properties of materials, which are displayed at different levels. For example, the viscosity of steel is small as compared with asphalts. Granular materials also display minimal viscosity, as they practically do not creep under constant loads. However, the intrinsic cyclic loading of saturated sand causes phenomena that resemble the creep behaviour. Figure 5 shows the deformations of initially dilative sand for similar effective stress path as the one in the previous section (Fig. 4). The only difference is the initial state of saturated sand. Recall that the initially contractive soil liquefies during the first cycle of porepressure changes. The behaviour of the initially dilative soil is different as it does not liquefy, but the deformations of samples resemble deviatoric creep. In the case considered, the volumetric strain remains almost constant after a small initial increase in volume, caused probably by technical imperfections during the experiment.

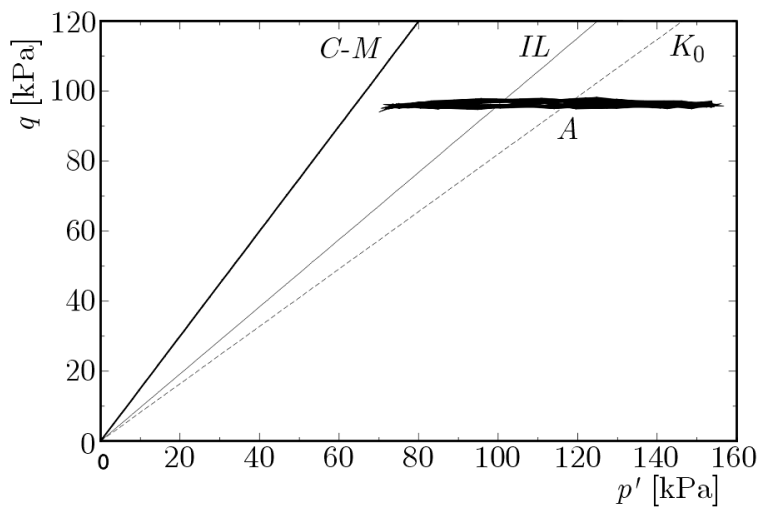

Fig. 4. Intrinsic cyclic loading at $q=95 \mathrm{kPa}$

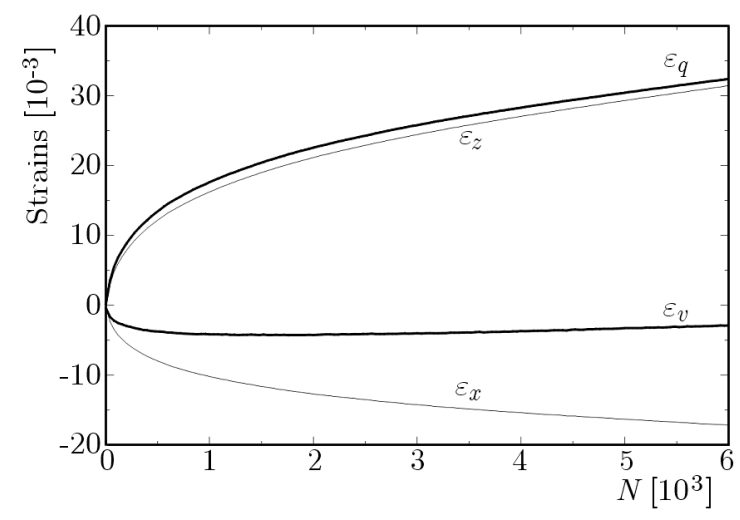

Fig. 5. Apparent deviatoric creep of saturated sand

Apparent deviatoric creep shown in Fig. 5 can be approximated by a simple power law, as follows

$$
\varepsilon_{q}=\alpha N^{\beta}
$$


where $\alpha$ and $\beta$ are parameters, and $N$ denotes the number of loading cycles, treated as a continuous variable. For the data from Fig. 5, one obtains $\alpha=1.594$ and $\beta=0.346$ for the strain unit $10^{-3}$, see Sawicki et al. (2013).

\section{Influence of the deviatoric stress}

The experiment shown in Fig. 5 has been performed at the constant deviatoric stress $q=95 \mathrm{kPa}$, the mean effective stress $p_{0}^{\prime}=115 \mathrm{kPa}$ and the amplitude of cyclic changes $\Delta p^{\prime}=40 \mathrm{kPa}$. In this case, the effective stress path cyclically crosses the instability line. Note that the vertical strain $\varepsilon_{z}$ is positive (vertical compression), and the horizontal strain $\varepsilon_{x}$ is negative (horizontal extension). Figure 7 shows the results of the experiment performed at a smaller deviatoric strain $q=45 \mathrm{kPa}$. The other stresses are as follows: $p_{0}^{\prime}=105 \mathrm{kPa}$ and $\Delta p^{\prime}=30 \mathrm{kPa}$ (Fig. 6 , path $B$ in Fig. 2). Both samples are initially dilative, and their initial void ratios are $e=0.562$ and 0.538 , respectively.

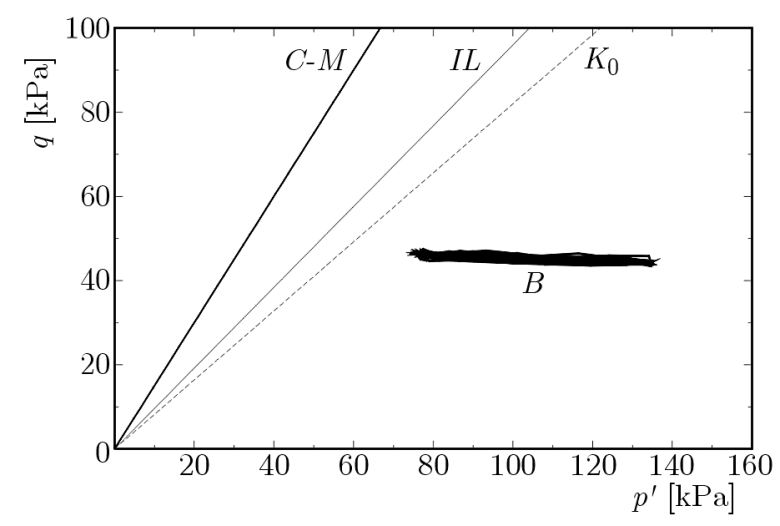

Fig. 6. Intrinsic cyclic loading at $q=45 \mathrm{kPa}$

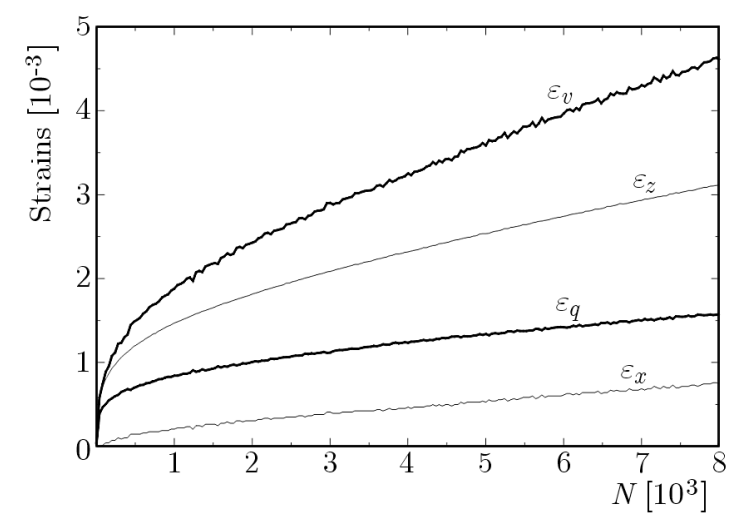

Fig. 7. Strains that developed during intrinsic cyclic loading at a smaller deviatoric stress, cf. Fig. 5

The basic difference between Figs. 5 and 7 is that the horizontal strain $\varepsilon_{x}$ is positive in the second case, i.e. the sample is horizontally compressed. The corresponding changes in the shapes of these samples are schematically shown in Fig. 8.

\section{Anisotropic effects}

Other interesting experiments involved intrinsic cyclic loading at $q=0$ (see Fig. 9, path $C$ in Fig. 2). Figure 10 shows the strains that developed for an effective stress path characterized by $p_{0}^{\prime}=100 \mathrm{kPa}$ and $\Delta p^{\prime}=35 \mathrm{kPa}$. The sample has been initially dilative $(e=0.557)$. 


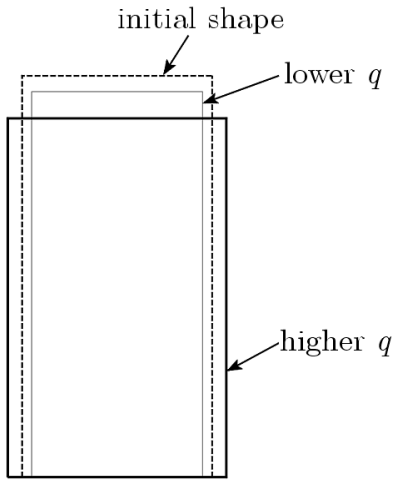

Fig. 8. The influence of the deviatoric stress level on the kind of deformation. Higher $q$ corresponds to Fig. 5, smaller to Fig. 7

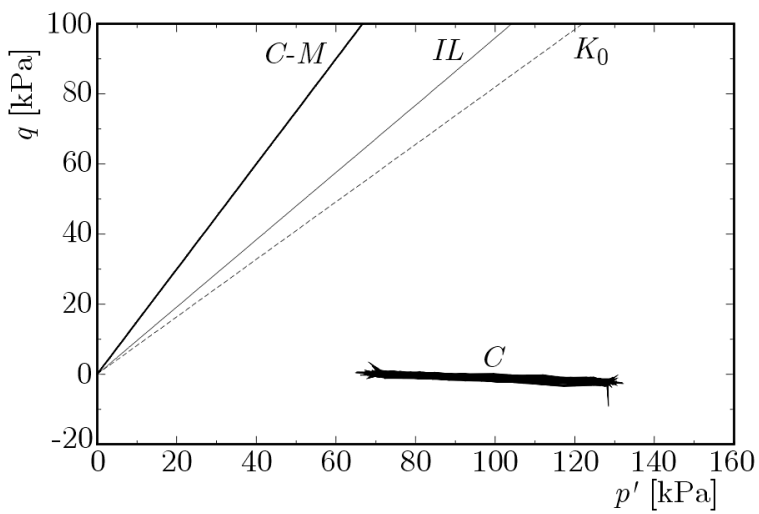

Fig. 9. Intrinsic cyclic loading at $q=0$

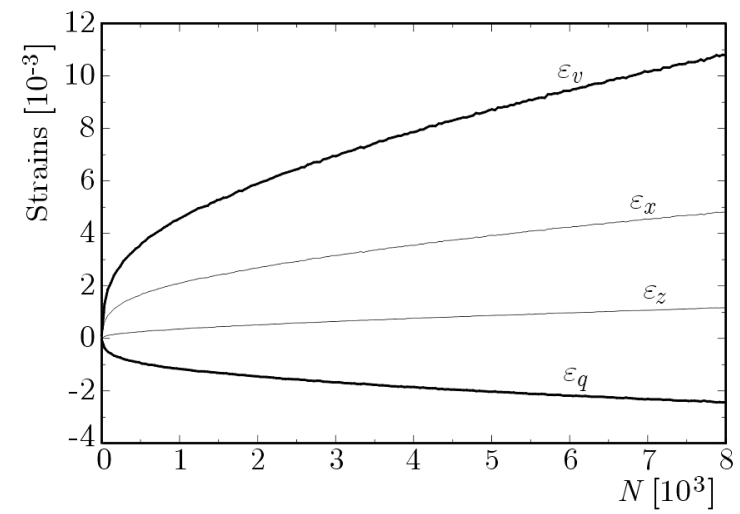

Fig. 10. Strains that developed during intrinsic cyclic loading at $q=0$

The most important observation is that isotropic intrinsic cyclic loading, without any deviatoric stress, causes permanent deformations of saturated sand, including an increasing deviatoric strain. In isotropic materials, changes in the spherical stress (the mean effective stress in this case) cause only volumetric deformations. Deviatoric strains appear only when the material is initially anisotropic. Figure 10 shows that the saturated sand tested is initially anisotropic, as $\varepsilon_{q}$ increases with the number of loading cycles. This effect is probably caused by the sample preparation methods which lead to the cross-isotropic structure of samples (the simplest case of anisotropy). Note that most geotechnical models do not take into account the effect of anisotropy. One more interesting observation is that the volumetric strain also permanently increases, which is another unexpected result. 
A similar effect of anisotropy has been observed during the external loadings of the soil specimen in the triaxial apparatus, see Sawicki and Świdziński (2010a). For example, during the isotropic loading of the triaxial specimen, the ratio of average volumetric and deviatoric strains is about 5 .

\section{Discussion and conclusions}

- The term intrinsic cyclic loading has been coined to describe forced cyclic pore-pressures changes in saturated granular soils. Such a mechanism appears in marine engineering, when changes in pore pressures are caused by water waves, see Jeng (2013). A different mechanism of pore-pressure changes occurs in most geotechnical problems in which the changes are caused by external excitations, such as earthquakes, machine vibrations, traffic, etc. In such cases, pore pressure is generated under undrained conditions, which leads to the macroscopic degradation of soil properties and to subsequent liquefaction. No cyclic changes in the excess pore pressure take place.

- Original experiments described in this paper have been performed to study the behaviour of saturated sand subjected to cyclic pore-pressure changes. Atypical effects observed in the experiments are presented in the subsequent sections. It should be noted first that intrinsic cyclic loading is isotropic, i.e. the mean effective stress changes cyclically, whereas the stress deviator remains constant. This type of loading causes a permanent increase in strains with the increasing number of loading cycles, contrary to the hitherto prevailing view that it causes only elastic cyclic changes in the volumetric strain and no changes in the deviatoric strain. In geotechnical engineering, the commonly accepted opinion is that the cyclic deviatoric stress leads to the compaction of drained sand or to liquefaction under undrained conditions.

- The response of saturated sand to intrinsic cyclic loading depends on its initial state. An initially contractive sample liquefies almost instantaneously, as shown in Fig. 3. The behaviour of initially dilative samples is different, since these samples do not liquefy but become permanently deformed as the number of loading cycles increases, see Figs. 5, 7 and 10. The character of these deformations depends on the initial deviatoric stress, cf. Figs. 5 and 7. The basic difference between these two cases are different signs of horizontal strains. Another interesting observation is the effect of anisotropy during intrinsic cyclic loading at $q=0$, see Fig. 10. If the material is initially isotropic, such loading should lead to volumetric deformations only, with zero deviatoric strain. The deviatoric strain that develops during such an experiment increases with the number of loading cycles, which means that the sample has been initially anisotropic. This effect is not taken into account in most geotechnical investigations.

- Certain experiments performed on saturated sand, such as the one shown in Fig. 5, produce an effect that resembles deviatoric creep. During this experiment, the volumetric strain remains constant, except for a small change dilation at the beginning of loading. It should be noted that Figs. 5, 7 and 10 illustrate the mean trends in strains. Small oscillations imposed on these trends have been omitted for clarity. Such oscillations correspond to the elastic response of the soil skeleton, which is not discussed in this paper.

- The investigations presented in this paper are experimental in nature. We have described some unusual phenomena observed during triaxial experiments with cyclic pore-pressure changes. Further experimental work in other laboratories is necessary to confirm or reject our findings. The experiments described may serve as a starting point for theoretical investigations. The problem described is important for the fundamental investigations in soil mechanics as well as in seabed mechanics. 
- The physical interpretation of the observed phenomena is difficult and needs further investigations. These are recently discovered phenomena, and their physical meaning needs a lot of further research. At present, we are not able to present the physical models of the presented phenomena.

\section{Acknowledgments}

Research reported in this paper was supported under project N N506 072938, financed by the Polish Ministry of Science and Higher Education, which is deeply appreciated.

\section{References}

1. Jefferies M., Been K., 2006, Soil Liquefaction, London, New York, Taylor and Francis

2. Jeng D.-S., 2013, Porous Models for Wave-seabed Interactions, Heidelberg, New York, Dordrecht, London, Springer and Shanghai Jiao Tong University Press

3. Lade P.V., Yamamuro J.A., Eds., 1999, Physics and Mechanics of Soil Liquefaction, Rotterdam, Brookfield, Balkema

4. SAWICKI A., 2011, Dilation and stability of sand in triaxial test, Geotechnical Engineering Journal of the SEAGS and AGSEA, 42, 2, 101-112

5. SAwicki A., 2013, Discussion of "Pore Pressure, Stress Distributions and Instantenous Liquefaction of Two-Layer Soil under Waves" by M.B. Can Ulker (2012), Journal of Waterway, Port, Coastal and Ocean Engineering, ASCE, 138, 6, 435-450, submitted

6. SAWicki A., Chybicki W., 2009, On accuracy of prediction of pre-failure deformations of granular soils, Computers and Geotechnics, 36, 6, 993-999

7. Sawicki A., Mierczyński J., 2006, Developments in modeling liquefaction of granular soils, caused by cyclic loads, Applied Mechanics Reviews, Transaction ASME, 59, 2, 91-106, doi: $10.1115 / 1.2130362$

8. Sawicki A., MierczyŃski J., Świdziński W., 2014, Apparent creep of saturated sand caused by intrinsic cyclic loading, Journal of Geotechnical and Geoenvironmental Engineering, ASCE, 140, 2, 06013002-1-06013002-3

9. Sawicki A., Staroszczyk R., 2008, Wave-induced stresses and pore pressures near a mudline, Oceanologia, 50, 4, 539-555

10. SAwicki A., Świdziński W., 2010a, Stress-strain relations for dry and saturated sands, Part I: Incremental model, Journal of Theoretical and Applied Mechanics, 48, 2, 309-328

11. SAWICKI A., ŚwidziŃSKI W., 2010b, Stress-strain relations for dry and saturated sands, Part II: Predictions, Journal of Theoretical and Applied Mechanics, 48, 2, 329-373

12. Yamamoto T., Koning H.L., Sellmeijer H., Hijum E. van, 1978, On the response of a poroelstic bed to water waves, Journal of Fluid Mechanicas, 87 (Part 1), 193-206 